\title{
Histopathological evaluation of rotation sutures for involutional entropion*
}

\author{
STUART R SEIFF,' MAN KIM,' AND EDWARD L HOWES JR \\ From the 'Departments of 'Ophthalmology and 'Pathology, University of California Medical Center, San \\ Francisco, USA
}

SUMMARY Rotation sutures are a popular method of temporarily correcting involutional entropion. The permanence of the procedure depends on a fibrotic scar along the suture tracks. Sutures which create generous scars would probably produce the most satisfactory results. In this study 4-0 chromic gut, nylon, and silk sutures were evaluated in rabbit lower eyelids for their ability to create histologically demonstrable fibrous tracks. All three sutures incited inflammatory cells and fibroblastic activity with collagen formation at two and four weeks. Residual scar tracks were observed at six weeks (two weeks after suture removal) also. Human specimens showed similar types of scar formation from the sutures. It was apparent that all three sutures create fibrotic scars along their tracks sufficient to correct, at least temporarily, involutional entropion.

Involutional entropion has been attributed to laxity of the lower eyelid retractors, horizontal laxity of the lower eyelid or enophthalmos, and overriding mobile preseptal orbicularis muscle..$^{1-3}$ Rotation sutures are are a popular method of dealing quickly and simply with involutional entropion. - $^{411}$

Variations of these techniques ${ }^{7-10}$ provide ways of dealing with two of the three generally proposed mechanisms for involutional entropion. The three horizontal mattress sutures are believed to create scar tracks which fix the overriding preseptal orbicularis muscle and reinforce the attachments of the lower eyelid retractors to the inferior tarsus. As a result the rotation suture repair of entropion is moderately successful, especially when considered as a temporary measure. Its limitations are probably related to the persistence of lower eyelid horizontal laxity and a weak suture track running from the retractors through the orbicularis to the skin. Chromic gut $^{9-11}$ and silk ${ }^{5610}$ sutures have been described in these techniques.

No studies have been previously performed to determine which sutures create adequate fibrotic scar

Presented at Ophthalmic plastic and reconstructive surgery - past, present, and future: a tribute to Crowell Beard, MD, 10-11 December 1987, San Francisco, CA.

Correspondence to Stuart R Seiff, MD, Ophthalmic Plastic and Reconstructive Surgery Service, Department of Ophthalmology, A-750, University of California Medical Center, San Francisco, CA 94143, USA. tracks and so probably the most satisfactory results. We have studied this question by examining reactions to non-absorbable silk, non-absorbable monofilament nylon, and absorbable chromic gut sutures in rabbit and human specimens.

\section{Materials and methods}

Pigmented rabbits were used for the preliminary part of this study. 4-0 Nylon, chromic, and silk sutures were placed in the lower eyelids of the pigmented rabbit in the manner of Feldstein. ${ }^{10}$ The sutures were tied under minimal tension so as to not create an irritating ectropion. They were left in place for two and four weeks. In addition the sutures were removed at four weeks in another specimen and the suture tracks allowed to heal for an additional two weeks. At the end of this time the tissue was examined histopathologically. The tissue was stained with haematoxylin and eosin and with a trichrome stain.

For the second portion of the study specimens of human tissue were examined. Rotation sutures were used for temporary relief in three patients with involutional entropion and horizontal laxity. The sutures were placed in horizontal mattress fashion deep into the fornix in an attempt to include the lower eyelid retractor attachments. They were then brought superiorly and anteriorly to a level just below the lashes. The double arms were placed approximately 
$3 \mathrm{~mm}$ apart and tied on the skin under sufficient tension to create a mild ectropion. Three sutures were placed in each lower eyelid. The sutures were left in place for two weeks while the patients awaited a more definitive Quickert procedure ${ }^{2312}$ with horizontal shortening. The horizontal shortening was performed in the segment of the eyelid which contained the lateral rotation suture. The inferior portion of the suture in the fornix was not included in the specimen. These segments were submitted for histopathological examination. The findings in these specimens are discussed below. Silk, chromic, and nylon sutures were again used.

\section{Results}

Each specimen was orientated with the anterior part of the lower eyelid to the left, posterior to the right, and superior and inferior aspects of the lid correspondingly.

\section{RABBIT SPECIMENS}

Two weeks after placement all three sutures (silk, chromic, and nylon) had created fibrous tracks coursing through orbicularis muscle from inferior posterior to superior anterior (Fig. 1 left). These suture tracks had active fibroblastic proliferation and collagen deposition. Lymphocytic infiltrates were noted as well (Fig. 1, right, Figs. 2, 3).

At four weeks the reactions to all three sutures were not significantly different from that seen at the two-week interval, though a somewhat more vigorous fibroblastic response with denser, more regular collagen formation was apparent. Epithelial cell ingrowth along the silk suture track was noted.

The residual collagen scar track could be identified in the six-week specimens (two weeks after suture removal) for all three sutures (Figs. 4-6). Areas of epithelial ingrowth were noted in the silk and nylon specimens (Figs. 4, 5). Trichrome staining of these

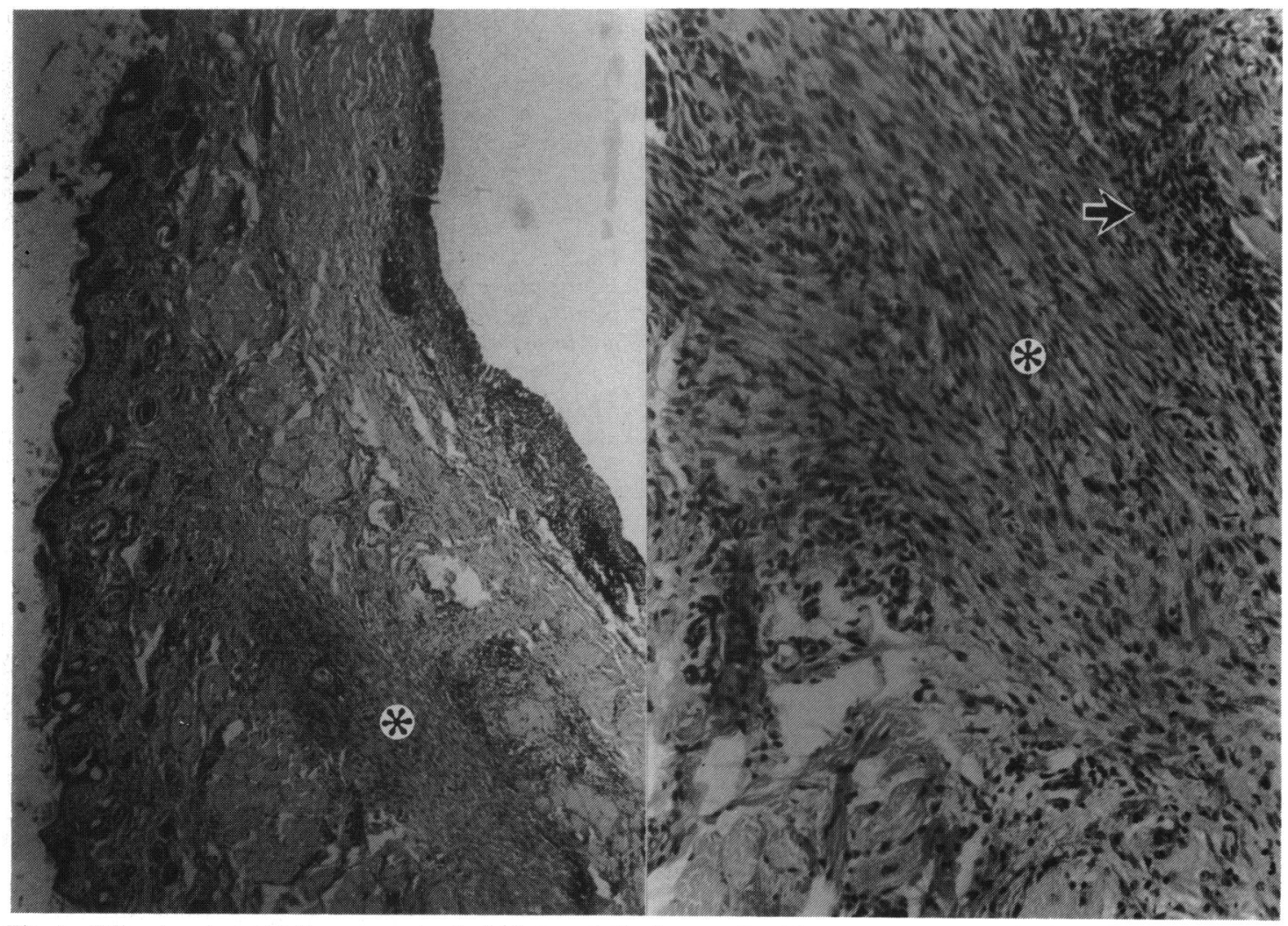

Fig. 1 Silk suture in rabbit for two weeks. Left: Suture track of active fibroblasts and collagen coursing through orbicularis muscle from inferior posterior to superior anterior eyelid (asterisk). (H and $E, \times 8$.) Right: Higher power view more clearly demonstrates the fibroblastic activity with collagen formation (asterisk). Some round chronic inflammatory cells are also seen (arrow). ( $H$ and $E, \times 33$.) 


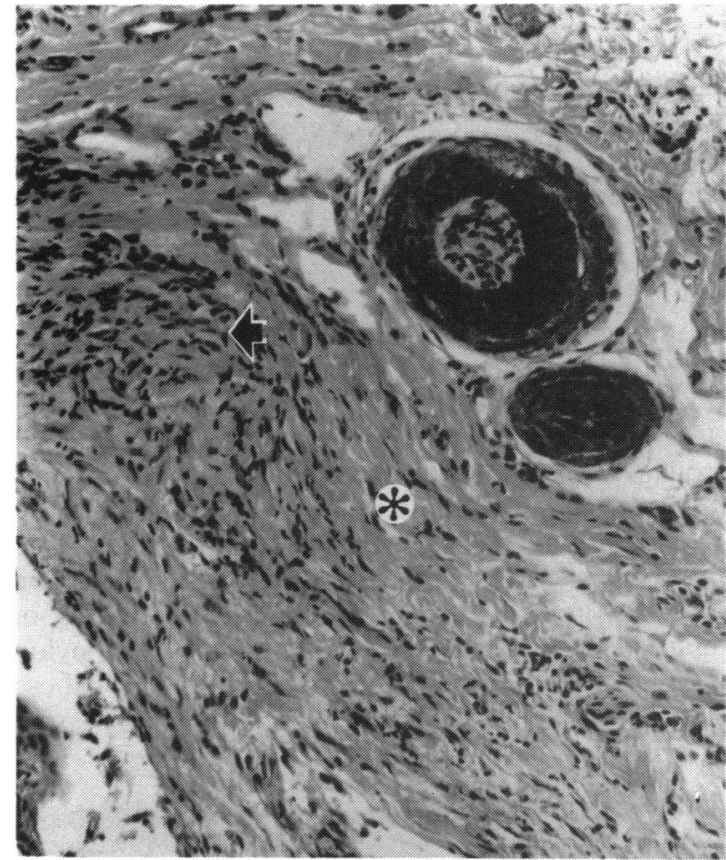

Fig. 2 Nylon suture in rabbit for two weeks. The suture track is composed of proliferating fibroblasts and collagen (asterisk) with chronic inflammatory cells (arrow). ( $H$ and $E, \times 32$.)

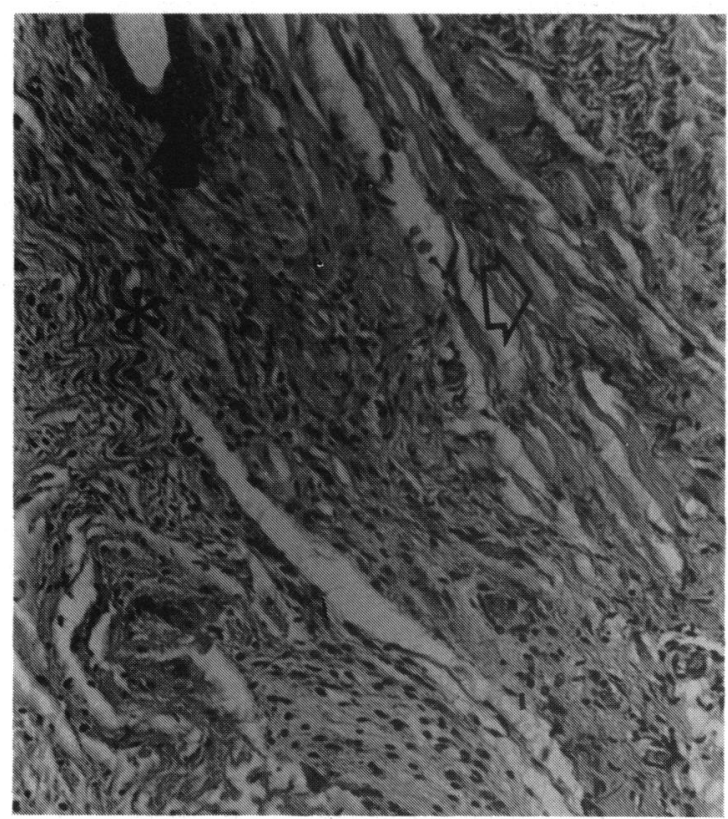

Fig. 4 Silk suture in rabbit, six-week specimen. The suture track is identified by fibroblasts and collagen (asterisk), atrophic orbicularis muscle fibres (hollow arrow), and epithelial cell ingrowth (solid arrow). ( $H$ and $E, \times 32$.)

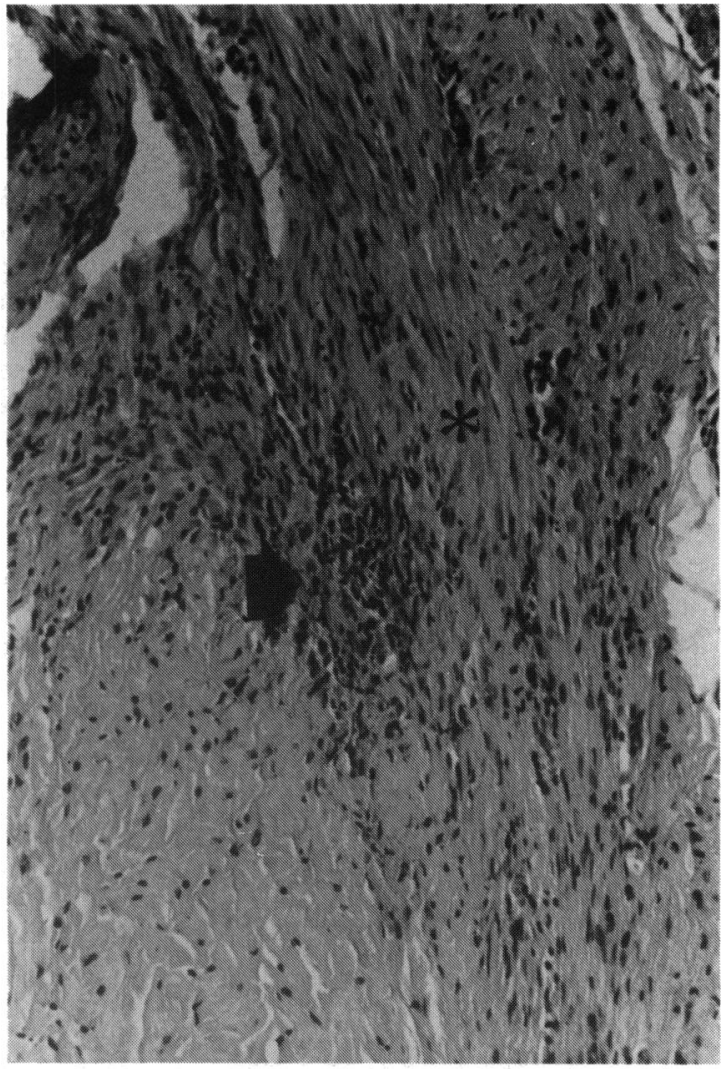

Fig. 3 Chromic gut suture in rabbit for two weeks.

Fibroblastic proliferation and collagen formation are

identifiable (asterisk) in the suture track. Inflammatory cells are noted (arrow). ( $H$ and $E, \times 32$ ).

six-week specimens better showed the substantial collagen present in the scar tracks.

\section{HUMAN SPECIMENS}

All human specimens were examined at two weeks. At this time the silk suture remnant was noted coursing through orbicularis muscle, surrounded by an intense epithelioid cell reaction (Fig. 7). The nylon suture specimen showed epithelial cell ingrowth along the suture with surrounding collagen formation. Chronic inflammatory cells were also seen (Fig. 8). The chromic gut suture track showed regular collagen formation by active fibroblasts along with chronic inflammatory cells (Fig. 9).

\section{Discussion}

Rotation sutures for entropion are best viewed as a temporary method for correction of the inturning 


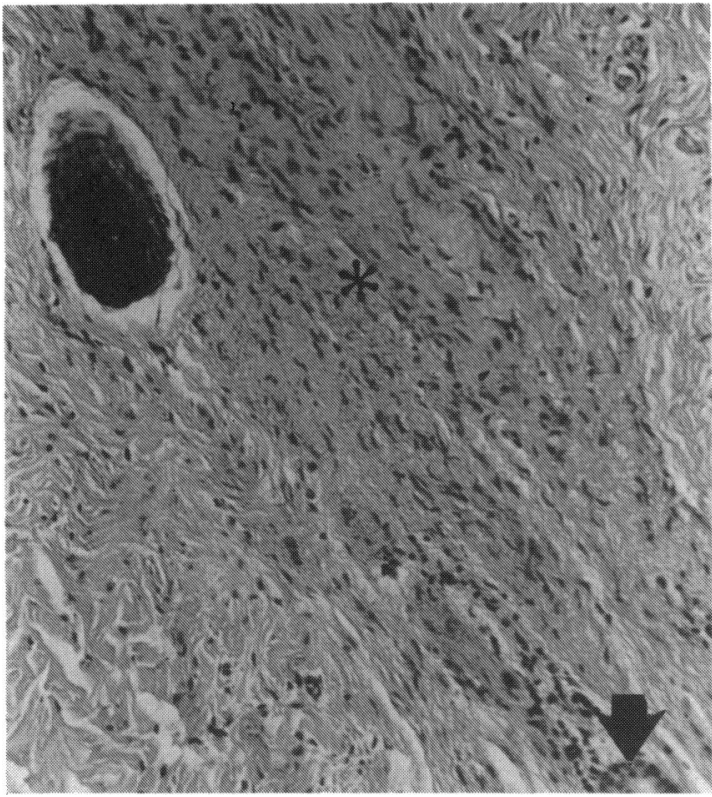

Fig. 5 Nylon suture in rabbit, six-week specimen. Collagen of the residual scar track appears well organised (asterisk) and a persistent area of epithelial ingrowth (arrow) is noted. ( $H$ and $E, \times 32$.)

lower eyelid margin until a more definitive procedure can be performed. After suture removal or dissolution the repair relies on the fibrotic track left behind. In this study both rabbit and human specimens were

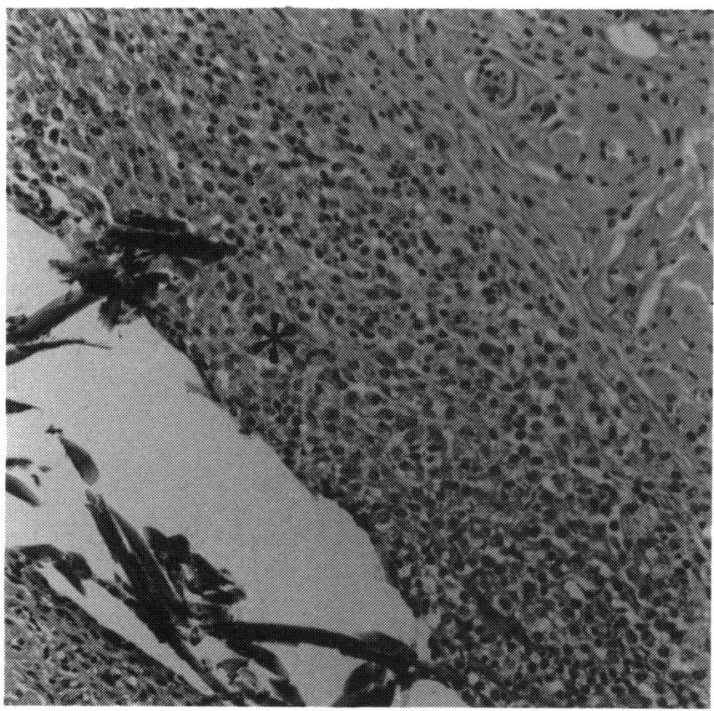

Fig. 7 Silk suture in patient for two weeks. The suture is surrounded by an intense epithelioid cell reaction (asterisk). ( $H$ and $E, \times 32$.)

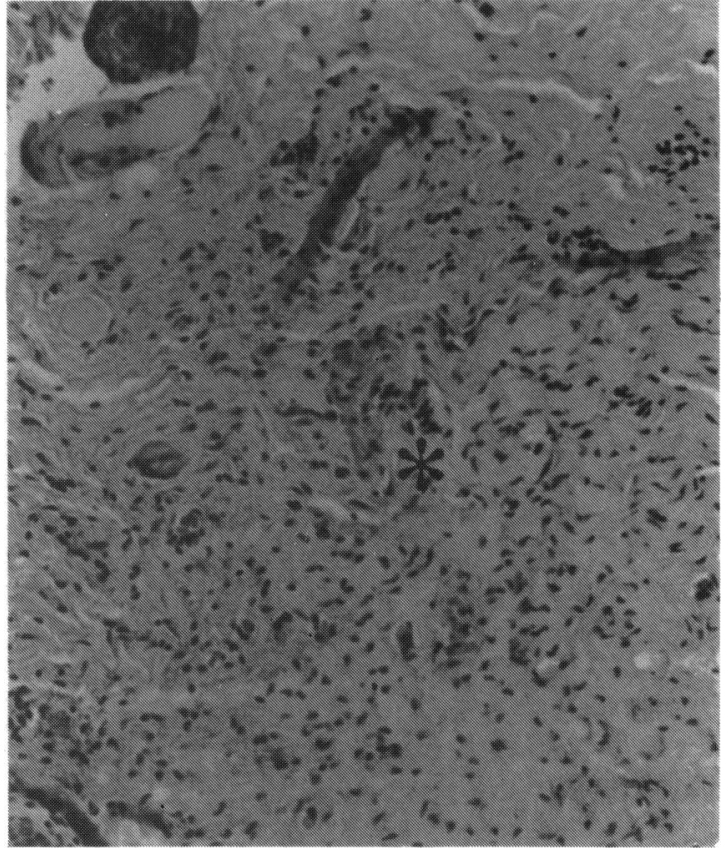

Fig. 6 Chromic gut suture in rabbit for six weeks. Fibroblasts and dense collagen (asterisk) are present within the scar track. ( $H$ and $E, \times 32$.)

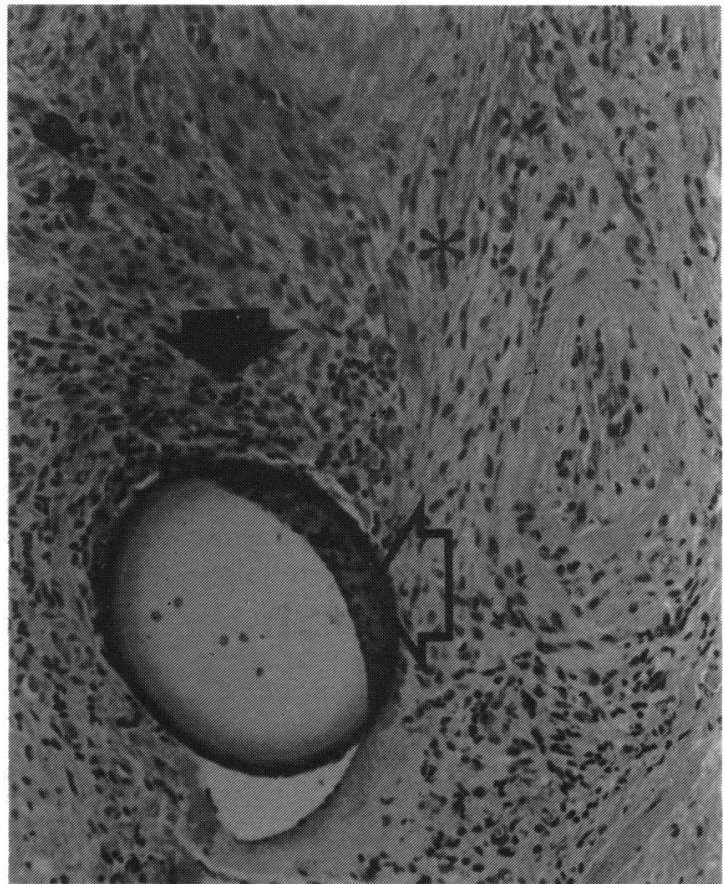

Fig. 8 Nylon suture in patient for two weeks. Collagen formation (asterisk), chronic inflammatory cells (solid arrow), and epithelial ingrowth (hollow arrow) around the suture are seen. ( $H$ and $E, \times 32$.) 


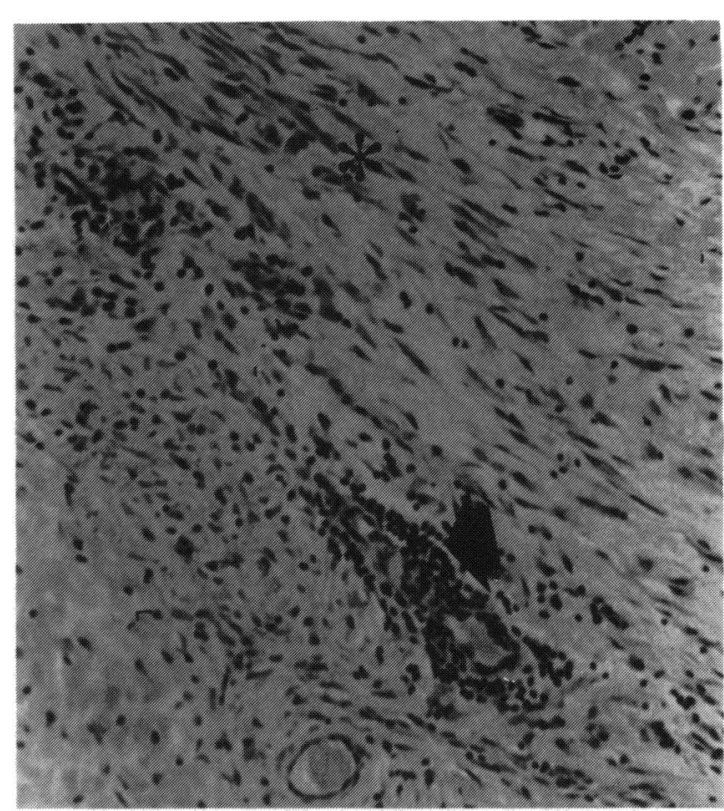

Fig. 9 Chromic gut suture in human for two weeks. Regular collagen formation by fibroblasts along the suture track is demonstrated (asterisk). Chronic inflammatory cells are evident (arrow). ( $H$ and $E, \times 32$.)

examined for scar track formation at varying intervals after suture placement.

Chromic gut, nylon, and silk sutures were studied in rabbit lower eyelids for their ability to create demonstrable fibrous tracks. All three sutures incited inflammatory reactions and fibroblastic activity with collagen formation at two and four weeks. Epithelial ingrowth was noted along the nylon and silk suture tracks as well. At six weeks residual scar tracks were observed for all three sutures.

In the human specimens, silk, nylon and chromic gut all provided an aggressive fibrotic and inflammatory response at two weeks.

In the treatment of involutional entropion an organised thick collagen track is desirable to reinforce the attachments between the inferior tarsus and the lower eyelid retractors and better to prevent overriding preseptal orbicularis. Clinically, silk rotation sutures are able, at least temporarily, to accomplish this. $^{5610}$

This study shows that nylon, chromic gut, and silk sutures all create histologically similar fibrotic scars along their tracks. The reasons for these fibrotic reactions are not clear. They could be due to an inflammatory response to the suture material itself or to tissue necrosis from suture tension. Whatever the reasons for the scar formation, the tracks should all be sufficient to correct an involutional entropion based on what we already know empirically about silk. Thus hypothetical differences in scar track formation should have a minimal role in the surgeon's choice of suture material. The availability of a doubled armed suture or the need for an absorbable material may in fact be more important considerations in any particular case.

\section{References}

1 Jones LT, Reeh MJ, Wobig JL. Senile entropion. Am J Ophthalmol 1972; 74: 327-9.

2 Collin JRO, Rathbun JE. Involutional entropion. Arch Ophthalmol 1978; 96: 1058-64.

3 Markovits AS. Variations on the theme of involutional entropion and the Quickert repair. Ann Ophthalmol 1980; 12: 1028-40.

4 Beard CH. Ophthalmic Surgery. 2nd ed. Philadelphia: Blakiston, 1914: 255-7.

5 Gaillard AL. Suture pour l'entropion. Ann Oculist (Paris) 1847; 18: 241 .

6 Arlt CF. Die Krankheiten des Auges für praktische Aertze geschildert. Prague: Credner, 1854; 3: 368.

7 Snellen H. Suture for entropion. Congress Internationale d'ophtalmologie (Paris), 1863: 236.

8 Stellwag von C. Ein neues Verfahren gegen einwartsgekehrte Wimptern. Allg Wien Med Zeitung 1893; 28: 527.

9 Illiff NT. An easy approach to entropion surgery. Ann Ophthalmol 1976; 8: 1343-6.

10 Feldstein M. Suture correction of senile entropion by inferior lid retractor tuck. Adv Ophthalmic Plastic Reconstr Surg 1983; 2: 269-74.

11 Quickert MH, Rathbun JE. Suture repair of entropion. Arch Ophthalmol 1971; 85: 304-5.

12 Quickert MH. In: Sorsby A, ed. Modern Ophthalmology. 2nd ed. London: Butterworth, 1972; 4: 937-54.

Accepted for publication 12 January 1989. 